\title{
Overview of jet physics with ALICE at the LHC
}

Mengliang Wang* for the AliCE Collaboration

\author{
Central China Normal University, Wuhan, China \\ SUBATECH, Ecole des Mines de Nantes, Université de Nantes, \\ CNRS-IN2P3, Nantes, France
}

\begin{abstract}
In these proceedings, a selection of recent results on jet physics from ALICE experiment is presented, including jet spectra, nuclear modification factors, jet structure and fragmentation observables, obtained in different collision systems ( $\mathrm{pp}, \mathrm{p}-\mathrm{Pb}$ and $\mathrm{Pb}-\mathrm{Pb}$ ).
\end{abstract}

\section{Physics motivations}

According to Lattice Quantum Chromodynamics, a phase transition from hadron gas to a deconfined phase of quarks and gluons, the Quark Gluon Plasma (QGP), is expected at high temperature or high baryon density. The QGP is studied experimentally using ultra-relativistic heavy-ion collisions.

ALICE (A Large Ion Collider Experiment) [1], one of the four large LHC experiments, is mainly dedicated to the study of the QGP properties. In the last six years, ALICE has collected a large amount of data. In this paper we will discuss results from proton-proton $(\mathrm{pp})$ and lead-lead $(\mathrm{Pb}-\mathrm{Pb})$ collisions at $\sqrt{s_{\mathrm{NN}}}=2.76 \mathrm{TeV}$ as well as from $\mathrm{p}-\mathrm{Pb}$ collisions at $\sqrt{s_{\mathrm{NN}}}=5.02 \mathrm{TeV}$. Measurements in pp collisions can be used as a test of perturbative QCD (pQCD) calculations as well as a baseline to quantify effects that may occur in more complex systems, such as proton-nucleus ( $\mathrm{p}-\mathrm{A}$ ) or nucleus-nucleus

\footnotetext{
*Email: mengliang.wang@subatech.in2p3.fr
} 
(A-A). p-A collisions are used to study possible Cold Nuclear Matter (CNM) effects, while A-A collisions are used to explore the properties of the QGP.

As the lifetime of the QGP is very short, its properties cannot be measured directly. Hard probes, such as jets (collimated showers of particles), are created at the early stage of the collision, before the QGP formation. The potential modification of jets, while they traverse and interact with the medium, can be used to probe the properties of the QGP. Comparing experimental results to theoretical models, quantities such as the transport coefficients can be extracted, providing information on the medium density or about energy loss mechanisms inside the QGP [2].

\section{How do we measure jets in ALICE?}

"Charged jets" are reconstructed using the ALICE mid-rapidity tracking system. The Inner Tracking System (ITS) and Time Projection Chamber (TPC) are used to measure charged particles down to very low transverse momentum $\left(p_{\mathrm{T}} \geq 150 \mathrm{MeV} / \mathrm{c}\right)$, in full azimuth and pseudo-rapidity $|\eta|<$ 0.9. To measure the electromagnetic component of a "full jet", clusters are reconstructed using the Electromagnetic Calorimeter (EMCal), also used as a trigger [3]. It covers $108^{\circ}$ in azimuth $\left(80^{\circ}<\phi<188^{\circ}\right)$ and $\sim 1.4$ units of rapidity $(|\eta|<0.67)$ with an energy resolution of $11 \% / \sqrt{E}+1.7 \%[1,3]$.

Jets have been reconstructed with different resolution parameters $R$ using the sequential recombination algorithm, anti- $k_{\mathrm{T}}$ from the FASTJET package, which has the advantage of being infrared and collinear safe [4].

\section{A selection of jet results from ALICE}

\subsection{Results from pp collisions}

In pp collisions at $7 \mathrm{TeV}$, ALICE has measured the differential "charged jet" production cross-sections for $20<p_{\mathrm{T}}^{\text {jet,ch }}<100 \mathrm{GeV} / c$ using several $R(R \in$ $[0.2,0.6])[5]$. Our measurements, shown for both $R=0.4$ and $R=0.6$ in Fig. 1, are in good agreement with the measurements from ATLAS [6].

The full jet cross-section has also been measured from $p_{\mathrm{T}}^{\text {jet }}=20$ to 120 $\mathrm{GeV} / c$ at $\sqrt{s}=2.76 \mathrm{TeV}[7]$. The corresponding result is shown on the right panel of Fig. 1 and is in good agreement with NLO pQCD calculations.

As an indirect measurement of the jet structure, ratios of the charged jet spectra obtained with $R=0.2,0.4$ and 0.6, are shown Fig. $2[5]$. For $p_{\mathrm{T}}^{\text {jet,ch }}>$ $30 \mathrm{GeV} / c$, those ratios are in good agreement with the ones obtained using 

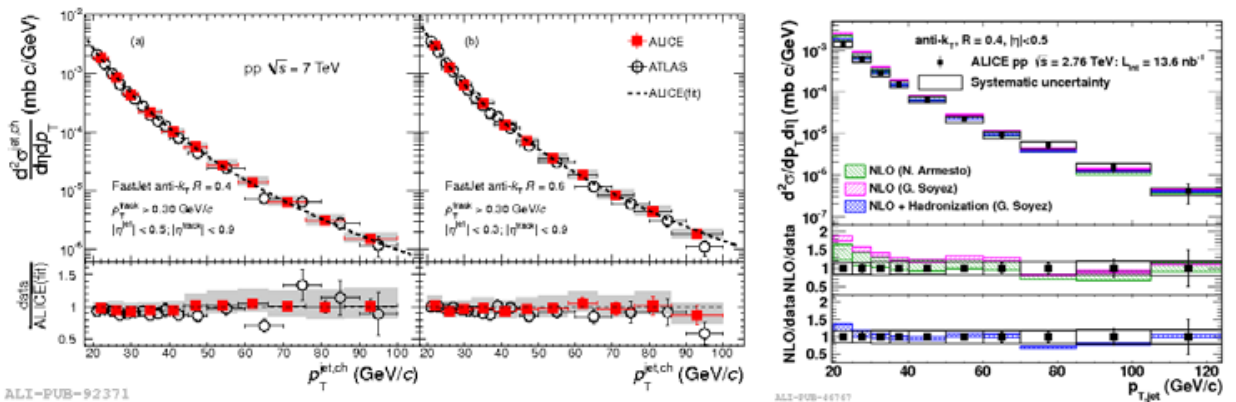

Figure 1: Left: Charged jet cross sections from ALICE (squares) and ATLAS (circles) in pp collisions at $7 \mathrm{TeV}$ for $R=0.4$ and 0.6. Right: Full jet $p_{\mathrm{T}}$ cross-section in pp collisions at $2.76 \mathrm{TeV}$ for $R=0.4$ compared to several $\mathrm{pQCD}$ predictions.

PYTHIA Perugia-2011 and HERWIG [8]. The distributions are increasing with jet $p_{\mathrm{T}}$ as the corresponding jets are more collimated. A similar study performed using full jets at $\sqrt{s}=2.76 \mathrm{TeV}$ shows that the data are in good agreement with NLO pQCD calculations including hadronization [7].
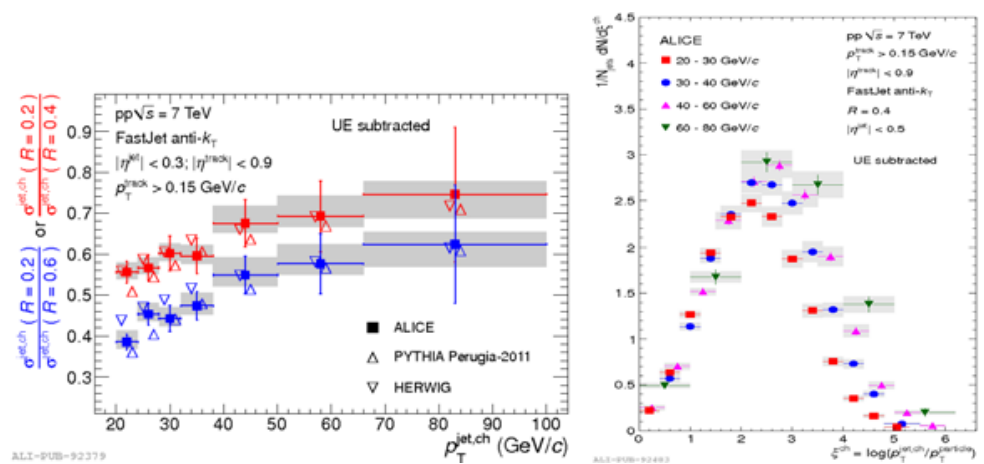

Figure 2: Left: Ratio of charged jet $p_{\mathrm{T}}$ cross-sections: $\sigma^{\text {jet,ch }}(R=0.2) / \sigma^{\text {jet,ch }}(R=$ $0.4)$ and $\sigma^{\text {jet,ch }}(R=0.2) / \sigma^{\text {jet,ch }}(R=0.6)$ (squares), compared with PYTHIA and HERWIG (triangles). Right: Particle $\xi$ distributions in leading charged jet.

In order to learn more about the internal structure of jets, ALICE has measured the distributions of the charged constituent transverse momentum in pp collisions [5]. The evolution of the measured leading jet fragmentation distributions with jet $p_{\mathrm{T}}\left(4\right.$ bins from $p_{\mathrm{T}}^{\text {jet,ch }}=20$ to $80 \mathrm{GeV} / c$ ) can be seen in Fig. 2 (right) as a function of $\xi=\log (1 / z)\left(z=p_{\mathrm{T}}^{\text {track }} / p_{\mathrm{T}}^{\text {jet,ch }}\right)$ and is in agreement with jet angular ordering expected from QCD color coherence [9].

\subsection{Results from $\mathrm{p}-\mathrm{A}$ collisions}

The $p_{\mathrm{T}}$-differential charged jet production cross-sections in $\mathrm{p}-\mathrm{Pb}$ collisions at $\sqrt{s}=5.02 \mathrm{TeV}$, for $R=0.2$ and 0.4 , and $20<p_{\mathrm{T}}^{\text {jet,ch }}<120 \mathrm{GeV} / c$, 
are in good agreement with boosted NLO pQCD calculations (taking into account the rapidity shift of the nucleon-nucleon center-of-mass system in $\mathrm{p}-\mathrm{Pb}$ with a boosted parton system) without and with nuclear modification of the Parton Distribution Function (nPDF) [10]. Within error bars the effect of nPDF can be neglected.

The ALICE charged jet $R_{\mathrm{pPb}}$ is presented in the left two panels of Fig. 3 [10]. Because of the lack of pp data at $\sqrt{s}=5.02 \mathrm{TeV}$, the $R_{\mathrm{pPb}}$ has been calculated using a pp reference which has been obtained by scaling the spectrum measured at $\sqrt{s}=7 \mathrm{TeV}$. The result is consistent with unity withing experimental uncertainties, which indicates the absence of strong Cold Nuclear Matter (CNM) effects on jet production.

The ratio of the charged jet cross-sections obtained with $R=0.2$ and 0.4 in $\mathrm{p}-\mathrm{Pb}$ collisions (Fig. 3 right panel), which does not show any strong centrality dependence [10], is compatible with the ratio obtained using $7 \mathrm{TeV}$ pp data (Fig. 2, left). The same behavior with respect to the collimation of jets thus applies for $\mathrm{p}-\mathrm{Pb}$ data. A good agreement with predictions from PYTHIA6 Perugia 2011 and POWHEG+PYTHIA8 simulations at $5.02 \mathrm{TeV}$ is also observed. Consequently, no significant energy dependence or change with collision species are observed. We observe no redistribution of the jet energy while comparing $R=0.2$ to $R=0.4$ in $\mathrm{p}-\mathrm{Pb}$ at $\sqrt{s_{\mathrm{NN}}}=5.02 \mathrm{TeV}$.
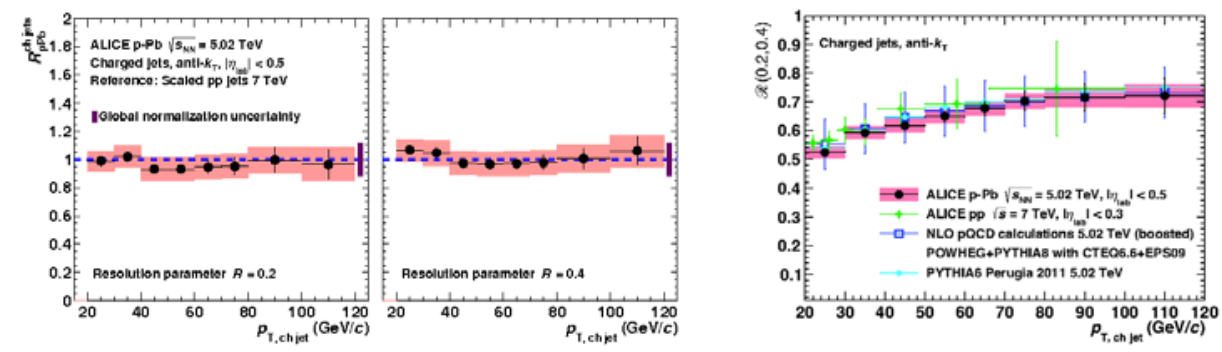

Figure 3: Left: Charged jet $R_{p A}$ in $\mathrm{p}-\mathrm{Pb}$ collisions at $5.02 \mathrm{TeV}$ measured using $R=0.2$ and 0.4 jets. Right: Ratio of the jet spectra $R=0.2 / R=0.4$ in $\mathrm{p}^{-}$ $\mathrm{Pb}$ collisions compared with results obtained using different collision systems or energies, and with PYTHIA Perugia 2011 and POWHEG+PYTHIA8 simulations.

\subsection{Results from $\mathrm{A}-\mathrm{A}$ collisions}

In $\mathrm{Pb}-\mathrm{Pb}$ collisions at $\sqrt{s_{\mathrm{NN}}}=2.76 \mathrm{TeV}$, the full jet $p_{\mathrm{T}}$ spectra and the corresponding $R_{\mathrm{AA}}$ (Fig. 4 left panels) have been measured using $R=0.2$, in the centrality bins $0-10 \%$ for $40<p_{\mathrm{T}}^{\text {jet }}<120 \mathrm{GeV} / c$ and, $10-30 \%$ for $30<$ $p_{\mathrm{T}}^{\text {jet }}<100 \mathrm{GeV} / c[11]$. A large suppression of the jet nuclear modification factor is observed in both cases. Since the jet $R_{\mathrm{pPb}}$ is consistent with unity 
(section 3.2), the observed suppression in $\mathrm{Pb}-\mathrm{Pb}$ is most likely due to final state effects: energy loss in the hot and dense medium.

The right panel of Fig. 4 presents a comparison of the measurements of jet $R_{\mathrm{AA}}$ at LHC (using $R=0.2$ ). For the results compared here, different jet reconstructions (full jets for ALICE, particle-flow jets for CMS and calorimeter jets ${ }^{\dagger}$ for ATLAS) and background subtraction methods are used, and the kinematics of the measurements are quite different [12]. However ALICE, CMS and ATLAS measurements are compatible within errors.
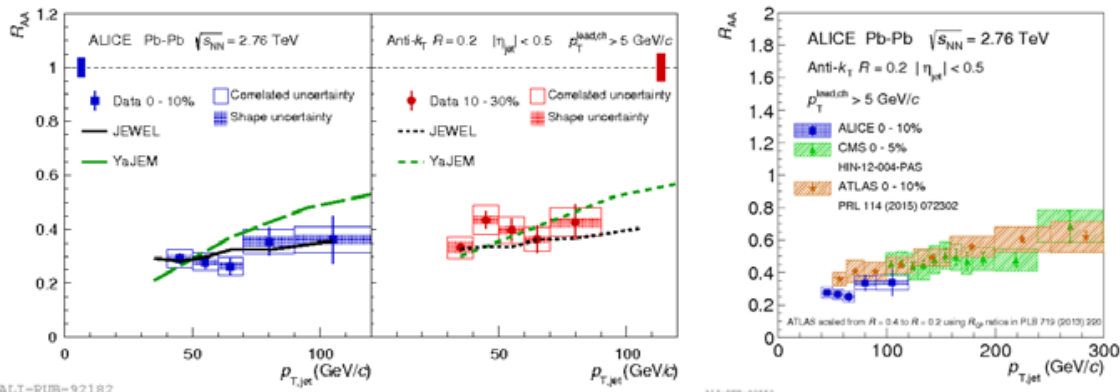

Figure 4: Left: $R_{\mathrm{AA}}$ of "full jets" measured in two centrality bins. Predictions from JEWEL and YaJEM are both in agreement with the data. Right: comparisons of the measured jet suppression at LHC (ALICE, CMS and ATLAS).

Doing such an inclusive jet measurement using jets with a larger resolution parameter $R$ would certainly be very interesting. However, in heavy ion collisions at LHC, jet measurements are affected by the large and fluctuating background, increasing with $R$ and centrality as discussed in [13].

In order to overcome those limitations, correlations between a high $p_{\mathrm{T}}$ trigger hadron and the corresponding recoil-jet can be used. Doing so, the parton producing the recoil-jet is biased toward higher in-medium path length, while the leading hadron is close to the surface of the fireball [14]. This analysis technique (at LHC, unique to ALICE) has several advantages: it provides us with a good handle on the combinatorial background which is statistically subtracted when computing the difference between two recoil-jet spectra, corresponding to different values of trigger hadron $p_{\mathrm{T}}$. It allows us to use jets with a larger $R$, without any fragmentation bias on the recoil-jet side. Recoil jet spectra were measured for $20<p_{\mathrm{T}}^{\text {jet,ch }}<100 \mathrm{GeV} / c$ using $R$ $=0.2,0.4$ and $0.5[15]$. The recoil jet yield is found to be suppressed when compared to reference pp distributions at $\sqrt{s}=2.76 \mathrm{TeV}$ using Monte-Carlo or NLO pQCD methods (validated using pp data at $\sqrt{s}=7 \mathrm{TeV}$ ). No signif-

\footnotetext{
${ }^{\dagger}$ The ATLAS $R_{A A}$ was scaled from $R=0.4$ to $R=0.2$ using their jet $R_{C P}$ ratio [12].
} 
icant evidence of intra-jet broadening nor of medium-induced acoplanarity are observed using jets with resolution parameters up to $R=0.5$.

\section{Outlook}

During the first LHC long shutdown, an extension of EMCal called Di-jet Calorimeter (DCal) was installed in ALICE. It will allow jet-jet and photonjet measurements while providing us with increased triggering capabilities. With the start of the LHC run 2, many new exciting results are expected.

\section{References}

[1] K. Aamodt et al. (ALICE Coll.), JINST 3 (2008) S08002; B. Alessandro et al. (ALICE Coll.), J. Phys. G 32 (2006) 1295

[2] M. Gyulassy and M. Plumer, Phys. Lett. B 243 (1990) 432

[3] U. Abeysekara et al. (ALICE EMCal Coll.), arXiv:1008.0413

[4] M. Cacciari, G. P. Salam and G. Soyez, Eur. Phys. J.C 72 (2012) 1896

[5] B. Abelev et al. (ALICE Coll.), Phys. Rev. D 91 (2015) 112012

[6] G. Aad et al. (ATLAS Coll.), Phys. Rev. D 84 (2011) 054001

[7] B. Abelev et al. (ALICE Coll.), Phys. Lett. B 722 (2013) 262

[8] PYTHIA: T. Sjostrand, S. Mrenna and P. Skands, JHEP 05 (2006) 026; HERWIG: G. Corcella et al., JHEP 01 (2001) 010

[9] B. Ermolaev and V. S. Fadin, JETP Lett. 33 (1981) 269. A. H. Mueller, Phys. Lett. B 104: (1981) 161

[10] J. Adam et al. (ALICE Coll.), Phys. Lett. B 749 (2015) 68

[11] J. Adam et al. (ALICE Coll.), Phys. Lett. B, 746 (2015) 1

[12] CMS HIN-12-004-PAS; G. Aad et al. (ATLAS Coll.), Phys. Rev. Lett. 114 (2015) 072302, Phys. Lett. B 719 (2013) 220

[13] B. Abelev et al. (ALICE Coll.), JHEP 03 (2012) 053

[14] G. De Barros et al., Nucl. Phys. A 910-911 (2013) 314

[15] J. Adam et al. (ALICE Coll.), JHEP 09 (2015) 170 